\title{
大气周日和半日迁移潮汐非线性传播及其 短期变化的数值研究
}

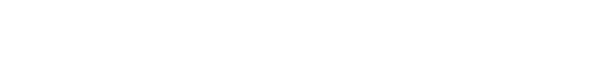 \\ (1) 武汉大学电子信息学院, 武汉 430079; (2) 武汉大学地球空间环境与大地测量教育部重点实验室, 武汉 430079. E-mai: zsd@whu.edu.cn)
}

\begin{abstract}
摘要 以全球尺度波模式(GSWM)给出的一月份周日和半日迁移潮汐线性稳态解作为初值, 采用三维 球坐标系下的全非线性动力学数值模式, 模拟了从地面到低热层的大气中, 周日和半日迁移潮汐的线 性和非线性传播, 并将模拟结果与 GSWM 和实验观测结果进行了定量比较. 研究结果表明: 受非线性 效应影响, 周日和半日潮汐在中、高层大气传播时其振幅会呈现明显的短期变化, 它们与背景大气的非 线性相互作用会显著改变全球中、高层大气的背景风场和温场, 这说明周日和半日潮汐的非线性传播对 中、高层背景大气的动力学和热力学暂态结构都有重要影响, 并且非线性效应是导致 GSWM 和观测结 果差异的重要原因.
\end{abstract}

关键词 周日迁移潮汐 半日迁移潮汐 非线性效应 中、高层大气

中、高层大气中关键的全球尺度动力学过程一般 都与大尺度波动密切相关. 大气潮汐是典型的大尺 度波动, 其振动周期为一个太阳日的整数分之一. 在 中、高层大气中，普遍存在着潮汐波和重力波两种大 气波动. 在中层大气中, 与潮汐相比, 重力波垂直波 长一般较小, 从而更容易被背景剪切风场吸收而无 法向上传播. 因此在更高的高度上, 即中层顶至低热 层(90 120 km)这一大气层区内, 潮汐波是占主导地 位的大气波动形式. 事实上大量的实验观测结果都 表明在中层顶和低热层大气中, 潮汐的全球传播对 该层区大气环流及热结构的形成都起着关键作用. 振动周期为一个和二分之一个太阳日的大气潮汐分 别被称为周日和半日潮汐. 其中, 随着太阳一起向西 传播的周日和半日潮汐分别被称为周日和半日迁移 潮汐, 它是由大气对太阳辐射的周期性吸收所激发. 为叙述简便，本文中将周日迁移潮汐简称为周日潮 汐，半日迁移潮汐简称为半日潮汐.

尽管大量的中、高层大气观测资料已为我们提供 了大气潮汐振幅和相位的空间以及季节变化的基本 特征，然而受探测设备的时、空间覆盖的限制，现有 的中、高层大气探测资料还不足以完整反映全球大气 潮汐的动力学特征. 因而, 仅仅利用观测数据来研究 大气潮汐是远远不够的. 为此人们在进一步加强实验 观测的同时, 开始关注于潮汐的理论和数值研究. 1970 年, Chapman和Lindzen ${ }^{[1]}$ 在线性近似条件下, 建 立了大气潮汐的基本理论, 目前这仍然是大气潮汐研
究的重要理论基础. 基于这一线性理论, 1995 年以来, Hagan等建立了一个全球大气潮汐模式(GSWM $)^{[2-4]}$, 这是迄今为止最具影响力的大气潮汐模式, 目前已被 广泛应用于研究大气潮汐的时空分布特征.

然而需要说明的是: 首先, 上述的潮汐理论和数 值模式本质上是线性的. 而在潮汐占主导地位的中 层顶和低热层高度上, 潮汐波的水平风振幅高达数 十 $\mathrm{m} / \mathrm{s}$, 甚至 $100 \mathrm{~m} / \mathrm{s}$, 这时非线性效应强烈, 完全不 满足线性近似的条件. 很多观测结果都表明在 $90 \mathrm{~km}$ 以上的高度上, 大气潮汐的振幅与 GSWM的计算结 果差异很大. 其次, 在不作线性近似的情况下, 要解 析甚至数值求解一个三维球坐标系下全非线性的大 气基本运动方程都是非常困难的, 再考虑到这个非 线性方程组中还包含了热激发和耗散等许多复杂的 物理过程, 解析求解就变得更加困难. 因此 1970 年 以来解析研究大气潮汐几乎没有任何重要进展. 这 限制了我们对潮汐的深入理解. 第三，尽管人们已经 认识到潮汐会与中层顶和低热层背景大气发生强烈 的非线性相互作用, 但是GSWM却无法提供这一相 互作用的任何信息, 这使得我们对关于潮汐波传播 导致的背景大气变化还缺乏定量的了解. 最后, GSWM给出的是一个大气潮汐的线性的稳态解, 在 其模式中完全无法反映潮汐的短期(时间尺度小于一 个波周期)变化. 而 $\mathrm{Yi}^{[5]}$ 在极区的观测研究表明, 大气 潮汐具有明显的短期变化特征. 总之, 要想更深入地 了解大气潮汐的短期变化以及伴随着潮汐传播的非 
线性效应, 必须利用一个全非线性的数值模式来模拟 潮汐的在中、高层大气中的传播. 最近, 在国际上也有 一些学者利用非线性的数值模式, 如 $\operatorname{GCM}($ General Circulation Model)模式对大气潮汐进行了研究 ${ }^{[6]}$, 但并 未涉及潮汐波的非线性传播与短期变化.

本文采用一个球坐标系下全非线性的数值模式 [7], 将GSWM给出的一月份潮汐稳态解作为初值, 首 次模拟了潮汐在中、高层大气中的全非线性传播过程. 本文先对我们采用的全非线性数值模式作了简单介 绍, 然后着重研究了周日和半日潮汐的短期(时间尺 度小于一个波周期)变化及非线性效应对背景大气暂 态结构的影响, 最后将模拟结果与 GSWM和实验观 测结果进行了定量比较, 分析了观测和GSWM结果 差异的原因.

\section{1 数值模式}

\section{1 控制方程}

从球坐标系下理想大气的基本运动方程出发, 考虑如下因素: (1) 中、高层大气的观测资料表明: 经 向和垂直方向的平均风一般明显小于纬向平均风, 因而在方程中我们只保留平均风场的纬向分量, 而 略去其经向和垂直分量. (2) 中、高层大气的观测和 不同数值模式的计算结果都说明背景大气参量(风场, 温度和密度等)在空间上主要随高度和纬度变化, 因 此在方程中我们略去背景大气参量的经度变化. 以 上简化方法是目前理论研究大气潮汐中所普遍采用 的. 这样三维球坐标系下的大气基本运动方程可以 写成如下形式:

$$
\begin{aligned}
& \frac{\partial u^{\prime}}{\partial t}+\frac{u_{0}+u^{\prime}}{a \cos \varphi} \frac{\partial u^{\prime}}{\partial \lambda}+\frac{v^{\prime}}{a} \frac{\partial\left(u_{0}+u^{\prime}\right)}{\partial \varphi}+w^{\prime} \frac{\partial\left(u_{0}+u^{\prime}\right)}{\partial r} \\
& -\frac{\left(u_{0}+u^{\prime}\right) v^{\prime}}{a} \tan \varphi+\frac{\left(u_{0}+u^{\prime}\right) w^{\prime}}{a} \\
& -2 \Omega \sin \varphi v^{\prime}+2 \Omega \cos \varphi w^{\prime} \\
& =-\frac{R}{a \cos \varphi}\left(\frac{\partial T^{\prime}}{\partial \lambda}+\frac{T_{0}+T^{\prime}}{\rho_{0}+\rho^{\prime}} \frac{\partial \rho^{\prime}}{\partial \lambda}\right) \\
& -D_{\lambda} u^{\prime}+\frac{1}{\rho_{0}} \frac{\partial}{\partial r}\left[\left(\mu_{0}+\rho_{0} v_{\text {eddy }}\right) \frac{\partial u^{\prime}}{\partial r}\right]-v_{R} u^{\prime}, \\
& \frac{\partial v^{\prime}}{\partial t}+\frac{u_{0}+u^{\prime}}{a \cos \varphi} \frac{\partial v^{\prime}}{\partial \lambda}+\frac{v^{\prime}}{a} \frac{\partial v^{\prime}}{\partial \varphi}+w^{\prime} \frac{\partial v^{\prime}}{\partial r}+\frac{2 u_{0} u^{\prime}+\left(u^{\prime}\right)^{2}}{a} \tan \varphi \\
& +\frac{v^{\prime} w^{\prime}}{a}+2 \Omega \sin \varphi u^{\prime}
\end{aligned}
$$

$$
\begin{aligned}
= & -\frac{R}{a}\left[\frac{\partial\left(T_{0}+T^{\prime}\right)}{\partial \varphi}+\frac{T_{0}+T^{\prime}}{\rho_{0}+\rho^{\prime}} \frac{\partial\left(\rho_{0}+\rho^{\prime}\right)}{\partial \varphi}-\frac{\partial T_{0}}{\partial \varphi}-\frac{T_{0}}{\rho_{0}} \frac{\partial \rho_{0}}{\partial \varphi}\right] \\
& -D_{\varphi} v^{\prime}+\frac{1}{\rho_{0}} \frac{\partial}{\partial r}\left[\left(\mu_{0}+\rho_{0} v_{\text {eddy }}\right) \frac{\partial v^{\prime}}{\partial r}\right]-v_{R} v^{\prime},
\end{aligned}
$$$$
\frac{\partial w^{\prime}}{\partial t}+\frac{u_{0}+u^{\prime}}{a \cos \varphi} \frac{\partial w^{\prime}}{\partial \lambda}+\frac{v^{\prime}}{a} \frac{\partial w^{\prime}}{\partial \varphi}+w^{\prime} \frac{\partial w^{\prime}}{\partial r}
$$$$
-\frac{2 u_{0} u^{\prime}+\left(u^{\prime}\right)^{2}+\left(v^{\prime}\right)^{2}}{a}-2 \Omega \cos \varphi u^{\prime}
$$$$
=-R\left[\frac{\partial\left(T_{0}+T^{\prime}\right)}{\partial r}+\frac{T_{0}+T^{\prime}}{\rho_{0}+\rho^{\prime}} \frac{\partial\left(\rho_{0}+\rho^{\prime}\right)}{\partial r}-\frac{\partial T_{0}}{\partial r}-\frac{T_{0}}{\rho_{0}} \frac{\partial \rho_{0}}{\partial r}\right]
$$$$
-D_{r} w^{\prime}+\frac{1}{\rho_{0}} \frac{\partial}{\partial r}\left[\left(\mu_{0}+\rho_{0} v_{\text {eddy }}\right) \frac{\partial w^{\prime}}{\partial r}\right]-v_{R} w^{\prime},
$$

$\frac{\partial \rho^{\prime}}{\partial t}+\frac{u_{0}+u^{\prime}}{a \cos \varphi} \frac{\partial \rho^{\prime}}{\partial \lambda}+\frac{v^{\prime}}{a} \frac{\partial\left(\rho_{0}+\rho^{\prime}\right)}{\partial \varphi}$

$$
+w^{\prime} \frac{\partial\left(\rho_{0}+\rho^{\prime}\right)}{\partial r}+\left(\rho_{0}+\rho^{\prime}\right)
$$$$
\times\left(\frac{1}{a \cos \varphi} \frac{\partial u^{\prime}}{\partial \lambda}+\frac{1}{a} \frac{\partial v^{\prime}}{\partial \varphi}+\frac{\partial w^{\prime}}{\partial r}\right)
$$

$$
+\left(\rho_{0}+\rho^{\prime}\right)\left(-\frac{v^{\prime}}{a} \tan \varphi+\frac{2 w^{\prime}}{a}\right)
$$

$=0$,

$$
\begin{aligned}
& \frac{\partial T^{\prime}}{\partial t}+\frac{u_{0}+u^{\prime}}{a \cos \varphi} \frac{\partial T^{\prime}}{\partial \lambda} \\
& +\frac{v^{\prime}}{a} \frac{\partial\left(T_{0}+T^{\prime}\right)}{\partial \varphi}+w^{\prime} \frac{\partial\left(T_{0}+T^{\prime}\right)}{\partial r} \\
& =\frac{(\gamma-1)}{R} J^{\prime}+\frac{(\gamma-1)}{R \rho_{0}} \\
& \frac{\partial}{\partial r}\left[\left(K_{0}+\rho_{0} K_{\text {eddy }}\right) \frac{\partial T^{\prime}}{\partial r}\right] \\
& -\alpha T^{\prime}-\frac{R\left(T_{0}+T^{\prime}\right)}{c_{v}} \\
& \left(\frac{1}{a \cos \varphi} \frac{\partial u^{\prime}}{\partial \lambda}+\frac{1}{a} \frac{\partial v^{\prime}}{\partial \varphi}+\frac{\partial w^{\prime}}{\partial r}-\frac{v^{\prime}}{a} \tan \varphi+\frac{2 w^{\prime}}{a}\right),
\end{aligned}
$$

其中, $t$ 为局地时间; $r(r=a+z)$ 是径向 (垂直)方向, 向上 为正; $a$ 为平均地球半径, 这里取 $a=6371 \mathrm{~km} ; z(0 \mathrm{~km}$ $\leq z \leq 128 \mathrm{~km})$ 是高度; $\lambda\left(0^{\circ} \leq \lambda \leq 360^{\circ}\right)$ 和 $\varphi\left(-90^{\circ} \leq \varphi \leq\right.$ $\left.90^{\circ}\right)$ 分别为经度和纬度; $u^{\prime}, v^{\prime}$ 和 $w^{\prime}$ 分别为东向、北向 和垂直方向扰动风速, 向东、向北和向上为正; $\rho^{\prime}$ 和 $T^{\prime}$ 分别为大气密度扰动和温度扰动; $u_{0}, \rho_{0}$ 和 $T_{0}$ 分别为 东向平均风、背景大气密度和温度; $R$ 为普适气体常 
数 $(R=286.9821 \mathrm{~N} \cdot \mathrm{m} / \mathrm{kg} \cdot \mathrm{K}) ; c_{v}$ 为定容比热 $\left(c_{v}=718\right.$ $\mathrm{J} / \mathrm{kg} \cdot \mathrm{K}), c_{p}$ 为定压比热 $\left(c_{p}=c_{v}+R\right), \gamma=C_{p} / c_{v} ; \Omega$ 为地球自 转角频率 $\left(\Omega=7.292 \times 10^{-5} \mathrm{Rad} \cdot \mathrm{s}^{-1}\right) ; \alpha$ 为牛顿冷却系数; $D_{\lambda}, D_{\rho}$ 和 $D_{\gamma}$ 分别代表纬向、经向和垂直方向的离子拽 力 ${ }^{[8]} ; v_{R}$ 为 Rayleigh摩擦系数; $J^{\prime}$ 为热激发函数; $K_{0}$ 为分 子热传导系数 $\left(K_{0}=0.015 T_{0}^{2 / 3} / M\right.$, 其中 $M$ 为大气平均 分子量), $\mu_{0}$ 为动力学分子稌性系数 $\left(\mu_{0}=0.266 K_{0} / R\right)$, $v_{\text {eddy }}$ 为运动学涡旋秥性系数, $K_{\text {eddy }}$ 为涡旋热传导系数 $\left(K_{\text {eddy }}=1.36 v_{\text {eddy }}\right)$.

需要指出的是(1)式是未作任何线性近似的, 所 有的非线性项都保留下来, 因而它是一个全非线性 的方程组. 本文中所提到的潮汐非线性传播解即是 我们对(1)式的计算结果. Hagan 等在建立 GSWM 的 过程中, 在(1)式的基础上作了线性近似, 即假定大 气潮汐是叠加在背景大气上的小扰动, 这样在略去 二阶小量的前提下, (1)式的线性化形式为:

$$
\begin{aligned}
& \frac{\partial u^{\prime}}{\partial t}+\frac{u_{0}}{a \cos \varphi} \frac{\partial u^{\prime}}{\partial \lambda}+\frac{v^{\prime}}{a} \frac{\partial u_{0}}{\partial \varphi} \\
& +w^{\prime} \frac{\partial u_{0}}{\partial r}-\frac{u_{0} v^{\prime}}{r} \tan \varphi-2 \Omega \sin \varphi v^{\prime} \\
& =-\frac{R}{a \cos \varphi}\left(\frac{\partial T^{\prime}}{\partial \lambda}+\frac{T_{0}}{\rho_{0}} \frac{\partial \rho^{\prime}}{\partial \lambda}\right)-D_{\lambda} u^{\prime} \\
& +\frac{1}{\rho_{0}} \frac{\partial}{\partial r}\left[\left(\mu_{0}+\rho_{0} v_{\text {eddy }}\right) \frac{\partial u^{\prime}}{\partial r}\right]-v_{R} u^{\prime} \\
& \frac{\partial v^{\prime}}{\partial t}+\frac{u_{0}}{a \cos \varphi} \frac{\partial v^{\prime}}{\partial \lambda}+\frac{2 u_{0} u^{\prime}}{a} \tan \varphi+2 \Omega \sin \varphi u^{\prime} \\
& =-\frac{R}{a}\left(\frac{\partial T^{\prime}}{\partial \varphi}+\frac{T_{0}}{\rho_{0}} \frac{\partial \rho^{\prime}}{\partial \varphi}-\frac{T_{0} \rho^{\prime}}{\rho_{0}{ }^{2}} \frac{\partial \rho_{0}}{\partial \varphi}+\frac{T^{\prime}}{\rho_{0}} \frac{\partial \rho_{0}}{\partial \varphi}\right)-D_{\varphi} v^{\prime} \\
& +\frac{1}{\rho_{0}} \frac{\partial}{\partial r}\left[\left(\mu_{0}+\rho_{0} v_{\text {eddy }}\right) \frac{\partial v^{\prime}}{\partial r}\right]-v_{R} v^{\prime}, \\
& \frac{\partial w^{\prime}}{\partial t}+\frac{u_{0}}{a \cos \varphi} \frac{\partial w^{\prime}}{\partial \lambda}-\frac{2 u_{0} u}{a}-2 \Omega \cos \varphi u^{\prime} \\
& =-R\left(\frac{\partial T^{\prime}}{\partial r}+\frac{T_{0}}{\rho_{0}} \frac{\partial \rho^{\prime}}{\partial r}-\frac{T_{0} \rho^{\prime}}{\rho_{0}{ }^{2}} \frac{\partial \rho_{0}}{\partial r}+\frac{T^{\prime}}{\rho_{0}} \frac{\partial \rho_{0}}{\partial r}\right)-D_{r} w^{\prime} \\
& +\frac{1}{\rho_{0}} \frac{\partial}{\partial r}\left[\left(\mu_{0}+\rho_{0} v_{\text {eddy }}\right) \frac{\partial w^{\prime}}{\partial r}\right]-v_{R} w^{\prime}, \\
& \frac{\partial \rho^{\prime}}{\partial t}+\frac{u_{0}}{a \cos \varphi} \frac{\partial \rho^{\prime}}{\partial \lambda}+\frac{v^{\prime}}{a} \frac{\partial \rho_{0}}{\partial \varphi}+w^{\prime} \frac{\partial \rho_{0}}{\partial r} \\
& +\rho_{0}\left(\frac{1}{a \cos \varphi} \frac{\partial u^{\prime}}{\partial \lambda}+\frac{1}{a} \frac{\partial v^{\prime}}{\partial \varphi}+\frac{\partial w^{\prime}}{\partial r}-\frac{v^{\prime}}{a^{s}} \tan \varphi\right)=0,
\end{aligned}
$$

$$
\begin{aligned}
& \frac{\partial T^{\prime}}{\partial t}+\frac{u_{0}}{a \cos \varphi} \frac{\partial T^{\prime}}{\partial \lambda}+\frac{v^{\prime}}{a} \frac{\partial T_{0}}{\partial \varphi}+w^{\prime} \frac{\partial T_{0}}{\partial r} \\
& =\frac{(\gamma-1)}{R} J^{\prime}+\frac{(\gamma-1)}{R \rho_{0}} \frac{\partial}{\partial r}\left[\left(K_{0}+\rho_{0} K_{\text {eddy }}\right) \frac{\partial T^{\prime}}{\partial r}\right] \\
& \quad-\alpha T^{\prime}-\frac{R T_{0}}{c_{v}}\left(\frac{1}{a \cos \varphi} \frac{\partial u^{\prime}}{\partial \lambda}+\frac{1}{a} \frac{\partial v^{\prime}}{\partial \varphi}+\frac{\partial w^{\prime}}{\partial r}-\frac{v^{\prime}}{a} \tan \varphi\right) .
\end{aligned}
$$

GSWM 的结果就是通过求解(2)式得到, 但是它 仅仅给出了(2)式月平均的稳态解, 即潮汐振幅和初 相位的空间分布, 而未考虑潮汐振幅的短期变化. 本 文中所提到的潮汐线性传播解是指我们对(2)式的计 算结果. 我们在求解(1)和(2)式的过程中均给出了潮 汐的传播解, 即考虑了潮汐振幅的短期变化. 另外, 在(1)和(2)式中, 热激发函数 $J^{\prime}$, 牛顿冷却系数 $\alpha$, 离 子拽力 $D_{\lambda}, D_{\rho}$ 和 $D_{\gamma}$, 运动学涡旋秥性系数 $v_{\mathrm{edd}}$, 以及 Rayleigh 摩擦系数 $v_{R}$ 都与 GSWM 中的取值一致.

\section{2 数值计算方法}

在数值计算(1)和(2)式的过程中, 我们选择不同 的方法来计算经向 $(\lambda)$ 、纬向 $(\varphi)$ 和垂直 $(r)$ 三个方向上 的空间微商: $\lambda$ 方向和 $\varphi$ 方向采用谱方法, $r$ 方向采用有 限差分法. 具体求解过程包括空间和时间差分方法, 边界条件的确定等在文献 [7]中已有详细描述, 在此仅介绍主要的计算参数. 我们在三个空间方向 上均采用均匀网格, 三个方向上的空间步长分别为, $\Delta \lambda=5^{\circ}, \Delta \varphi=3^{\circ}$ 和 $\Delta r=2 \mathrm{~km}$, 这与 GSWM 的精度相当. 时间步长的选择应满足如下的柯朗条件

$$
\Delta t<\Delta t_{c}=\frac{1}{\left(\mathrm{v}_{a}+v_{T}\right)\left[\frac{1}{\Delta r^{2}}+\frac{1}{(r \cos \varphi \Delta \lambda)^{2}}+\frac{1}{(r \Delta \varphi)^{2}}\right]^{\frac{1}{2}}},
$$

式中, $v_{\mathrm{a}}$ 为声速, $v_{T}=\sqrt{\left(u_{0}+u^{\prime}\right)^{2}+\left(v^{\prime}\right)^{2}+\left(w^{\prime}\right)^{2}}$. 由于 在实际计算中, 时间步长短有利于提高计算精度, 因 此, 在本文的计算中取 $\Delta t=0.25 \Delta t_{c}$.

\section{3 周日和半日迁移潮汐初值和计算结果处理}

周日迁移潮汐变量 $f_{\mathrm{DT}}=\left[u_{\mathrm{DT}}, v_{\mathrm{DT}}, w_{\mathrm{DT}}, \rho_{\mathrm{DT}}, T_{\mathrm{DT}}\right]$, 具有 $f_{\mathrm{DT}}=f^{\prime}{ }_{\mathrm{DT}} e^{i(\Omega t+\lambda)}$ 的形式, 其中 $\boldsymbol{f}_{\mathrm{DT}}^{\prime}=\hat{\boldsymbol{f}}_{\mathrm{DT}} \exp \left(i \boldsymbol{a}_{\mathrm{DT}}\right)$. $\hat{\boldsymbol{f}}_{\mathrm{DT}}=\left[\hat{u}_{\mathrm{DT}}, \hat{v}_{\mathrm{DT}}, \hat{w}_{\mathrm{DT}}, \hat{\rho}_{\mathrm{DT}}, \hat{T}_{\mathrm{DT}}\right]$ 和 $\boldsymbol{\alpha}_{\mathrm{DT}}=\left[\hat{u}_{\alpha \mathrm{DT}}\right.$, $\left.\hat{v}_{\alpha \mathrm{DT}}, \hat{w}_{\alpha \mathrm{DT}}, \hat{\rho}_{\alpha \mathrm{DT}}, \hat{T}_{\alpha \mathrm{DT}}\right]$ 分别是周日潮汐的振幅和 相位. 同样, 半日迁移潮汐变量 $f_{\mathrm{ST}}=\left[u_{\mathrm{ST}}, v_{\mathrm{ST}}\right.$, $\left.w_{\mathrm{ST}}, \rho_{\mathrm{ST}}, T_{\mathrm{ST}}\right]$ 具有 $\boldsymbol{f}_{\mathrm{ST}}=\boldsymbol{f}_{\mathrm{ST}}^{\prime} e^{i 2(\Omega t+\lambda)}$ 的形式, 其中 
$\boldsymbol{f}_{\mathrm{ST}}^{\prime}=\hat{\boldsymbol{f}}_{\mathrm{ST}} \exp \left(i \alpha_{\mathrm{ST}}\right), \hat{\boldsymbol{f}}_{\mathrm{ST}}=\left[\hat{u}_{\mathrm{ST}}, \hat{v}_{\mathrm{ST}}, \hat{w}_{\mathrm{ST}}, \hat{\rho}_{\mathrm{ST}}, \hat{T}_{\mathrm{ST}}\right]$ 和 $\boldsymbol{\alpha}_{\mathrm{ST}}=\left[\hat{u}_{\alpha \mathrm{ST}}, \hat{v}_{\alpha \mathrm{ST}}, \hat{w}_{\alpha \mathrm{ST}}, \hat{\rho}_{\alpha \mathrm{ST}}, \hat{T}_{\alpha \mathrm{ST}}\right]$ 分别是半日潮 汐的振幅和相位.

GSWM 给出的是月平均的潮汐稳态解, 在此我 们仅以一月份的结果为例进行分析. 将 GSWM 给出 的一月份周日或半日潮汐稳态解实部即 $\hat{\boldsymbol{f}}_{\mathrm{DT}} \cos \left(\lambda+\boldsymbol{\alpha}_{\mathrm{DT}}\right)$ 或 $\hat{\boldsymbol{f}}_{\mathrm{ST}} \cos \left(2 \lambda+\boldsymbol{\alpha}_{\mathrm{ST}}\right)$ 作为初值代入(1) 和(2)式进行计算, 可分别给出周日或半日潮汐非线 性和线性传播解.

为了从数值模拟结果中提取潮汐振幅信息, 我 们将所得到的计算结果 $\boldsymbol{g}^{\prime}(\lambda, \varphi, r, t)=\left[u^{\prime}, v^{\prime}, w^{\prime}, \rho^{\prime}, T^{\prime}\right]$ 按变量 $\lambda$ 进行如下谐波拟合

$$
\begin{gathered}
\boldsymbol{g}^{\prime}(\lambda, \varphi, r, t)=\boldsymbol{g}_{0}(\varphi, r, t)+\boldsymbol{g}_{1}(\varphi, r, t) \\
\cdot \cos \left[n \Omega t+n \lambda+g_{2}(\varphi, r, t)\right]
\end{gathered}
$$

当 $n$ 取 1 或 2 时便可分别得到周日或半日潮汐导致的 大气背景变化 $\left(g_{0}(\varphi, r, t)\right)$ 、周日或半日潮汐振幅 $\left(g_{1}(\varphi, r, t)\right)$ 和相位 $\left(g_{2}(\varphi, r, t)\right)$ 的纬度、高度分布和时 间演变.

\section{2 计算结果}

图 1 为 GSWM 给出的一月份周日和半日潮汐东 向风振幅的等值线图. 等值线的最小值为 $1 \mathrm{~m} / \mathrm{s}$, 振 幅大于 $5 \mathrm{~m} / \mathrm{s}$ 后，等值线之间的间隔为 $5 \mathrm{~m} / \mathrm{s}$.

\section{1 周日潮汐}

图 2 是采用我们的数值模式计算出的周日潮汐
在中、高层大气中传播了 $18 \mathrm{~h}$ 后的结果. 图 2(a)是采 用线性模式的计算结果, 图 2(b)是非线性传播的计算 结果. 由图 2(a)可见, 在线性情况下, 经过了 $18 \mathrm{~h}$ 的 传播, 潮汐振幅的纬度和高度分布与图 1(a)所示的初 值几乎完全一样, 它们都显示出周日潮汐在极区和 中纬地区的 90 120 km 处振幅较大, 而在其他区域 振幅较小的特点. 这说明我们的数值计算格式是可 靠的.

图 2(b) 是在非线性环境下, 周日潮汐在大气中传 播了 $18 \mathrm{~h}$ 后振幅的纬度和高度分布. 这是首次给出 周日潮汐在中、高层大气中的全非线性传播结果. 通 过比较图 1(a)和图 2(b)可以发现, 它们之间有明显的 差异, 特别是在波振幅较大的区域. 这说明在非线性 环境下, 周日潮汐会呈现明显的短期变化, 显然这一 变化来自于非线性效应的作用. 这一非线性效应可 能是导致潮汐观测结果和 GSWM 有明显差异的重要 原因.

为了更加清楚地观察非线性效应对东向风潮汐 振幅的影响, 图 3 给出了周日潮汐非线性传播与线性 传播下两者的差，即非线性传播下周日潮汐东向风 振幅(图 2(b))减去线性传播下东向风振幅(图 2(a)). 图中实线表示正值，虚线表示负值，等值线间的间隔 为 $1 \mathrm{~m} / \mathrm{s}$. 由于在 $60 \mathrm{~km}$ 以下, 大气潮汐振幅很小, 非 线性效应不明显, 线性解和非线性解的差异很小, 因
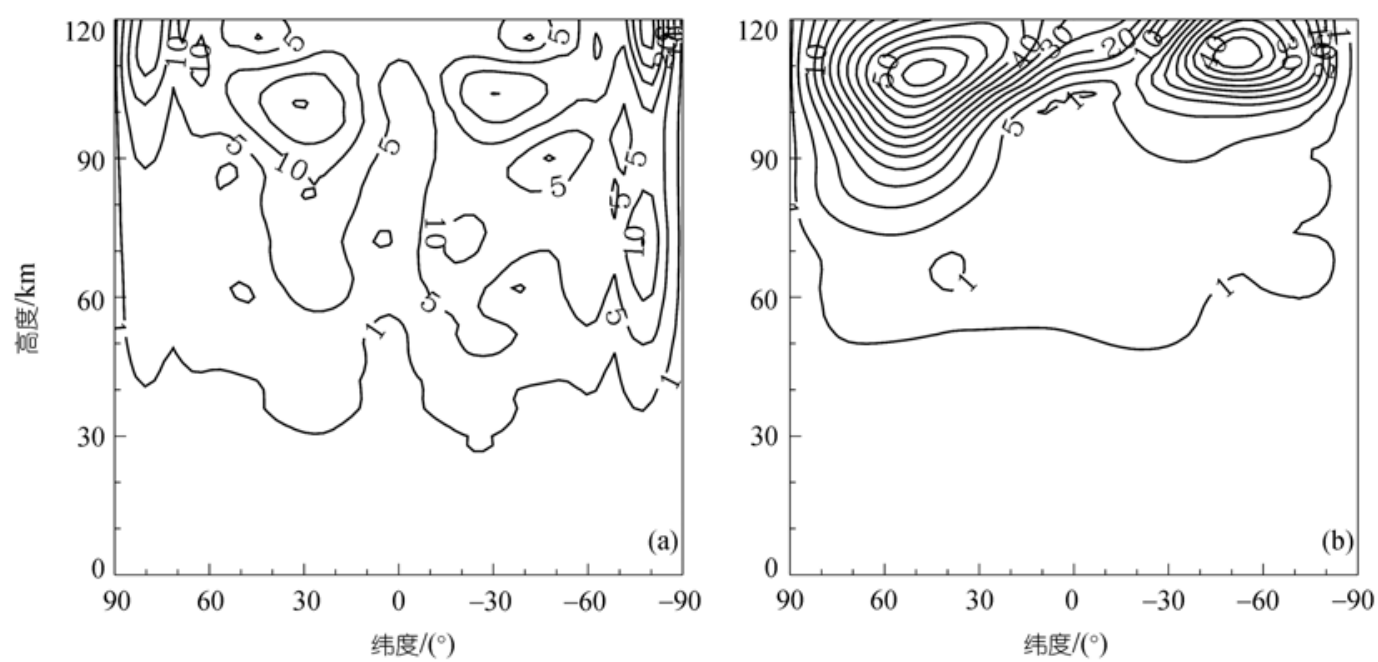

图 1 GSWM 给出的一月份迁移潮汐东向风振幅的纬度和高度分布 (a) 周日潮汐; (b)半日潮汐60/972004-687 

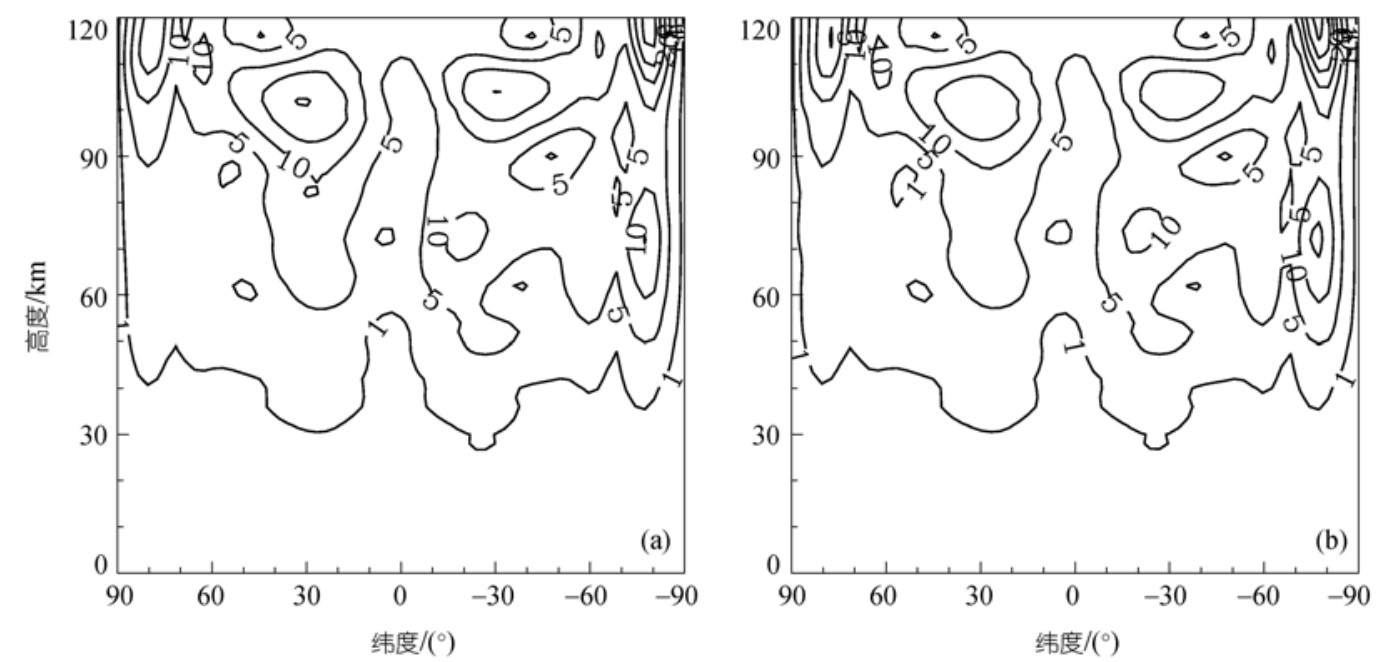

图 2 传播了 $18 \mathrm{~h}$ 后, 周日潮汐东向风分量振幅的纬度和高度分布

（a) 线性传播解; (b) 非线性传播解

而图 3 只给出了 60 120 km 高度范围内的结果. 由图 3 可见, 非线性效应使东向风潮汐振幅在有的区域增 加, 有的区域减小, 并且潮汐振幅(图 1(a)所示)越大、 非线性效应越强的区域, 这种差异也越大. 最大的差 异可达到 $10 \mathrm{~m} / \mathrm{s}$, 出现在 $120 \mathrm{~km}$ 高度上的南纬 $78^{\circ}$ 附近, 恰好是东向风潮汐振幅的最大值 (图 1(a) 所示) 处. 另一个差异明显的区域位于 $112 \mathrm{~km}$ 高度上的北 纬 $75^{\circ}$ 附近, 也正好是东向风潮汐振幅的一个极大值 处. 这些结果说明, 非线性效应是导致大气潮汐短期 变化的重要原因, 它对大气潮汐的全球结构有显著 影响, 从而提示我们在中间层和低热层中研究大气 潮汐时, 非线性效应是不可忽略的.

在线性环境下，潮汐波与背景之间不会发生相 互作用, 因此背景大气动力学和热结构都不会发生 显著变化. 而在非线性情况下, 潮汐波与背景大气的 非线性相互作用却会改变背景大气的动力学和热结 构, 这一非线性相互作用过程及其效应虽已被广泛 接受, 但迄今为止尚缺乏相关的定量研究. 图 4 给出 了周日潮汐经过 $18 \mathrm{~h}$ 非线性传播后, 导致背景东向 风的变化量的纬度和高度分布, 即(3)式中的 $g_{0}(\varphi, r$, $t)$. 图中, 实线代表正值(即波导致的背景风场增强), 虚线代表负值(即波导致的背景风场减弱)，等值线间 的间隔为 $1 \mathrm{~m} / \mathrm{s}$. 从图 4 可以观察到, 在 $90 \sim 120 \mathrm{~km}$ 的 高度范围内, 周日潮汐和背景的非线性相互作用对 几乎所有纬度上的背景风场都有影响. 在赤道和低 纬地区, 它会对东向风背景风场产生一个减速效应, 相反，在中纬地区会对背景风场产生加速，而在高纬

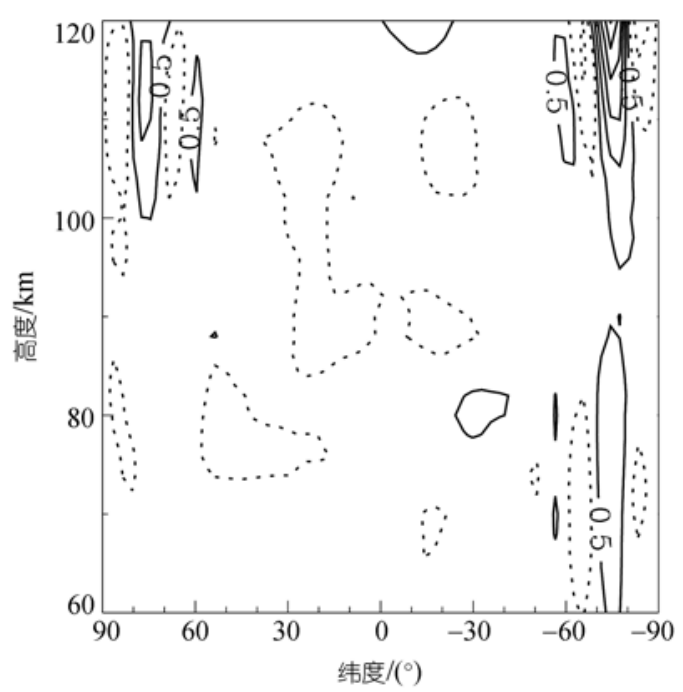

图 3 传播了 $18 \mathrm{~h}$ 后, 非线性传播与线性传播下周日潮汐 东向风振幅的差

地区，则交替出现减速和加速区域. 在极区，周日潮 汐波导致背景风场的改变最大可达 $7.5 \mathrm{~m} / \mathrm{s}$, 即在一 月份周日潮汐波对背景的平均加速度最大可达约 10 $\mathrm{m} \cdot \mathrm{s}^{-1} \cdot \mathrm{d}^{-1}$.

为了研究潮汐的非线性传播对中、高层大气热力 学结构的影响, 我们也给出了周日潮汐波导致的背 景温度变化(图 5). 图中, 实线代表正值(即波导致的 背景增温), 虚线代表负值(即波导致的背景降温), 等 值线间的间隔为 $1 \mathrm{~K}$. 由图 5 可见, 周日潮汐和背景 的非线性相互作用会对赤道地区的背景产生明显的 增温作用, 而对中 6 低纬地区的背景大气产生降温作 


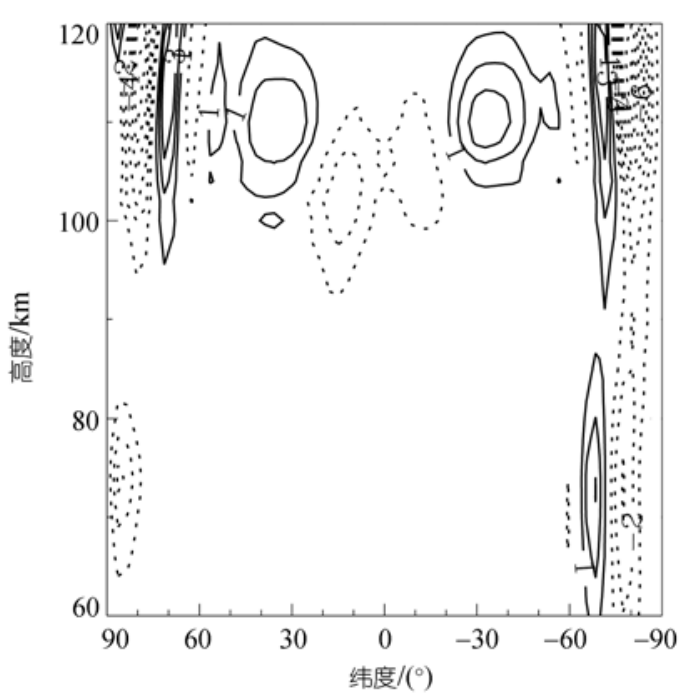

图 4 经过了 $18 \mathrm{~h}$ 的非线性传播后, 周日潮汐波导致的东 向背景风场变化

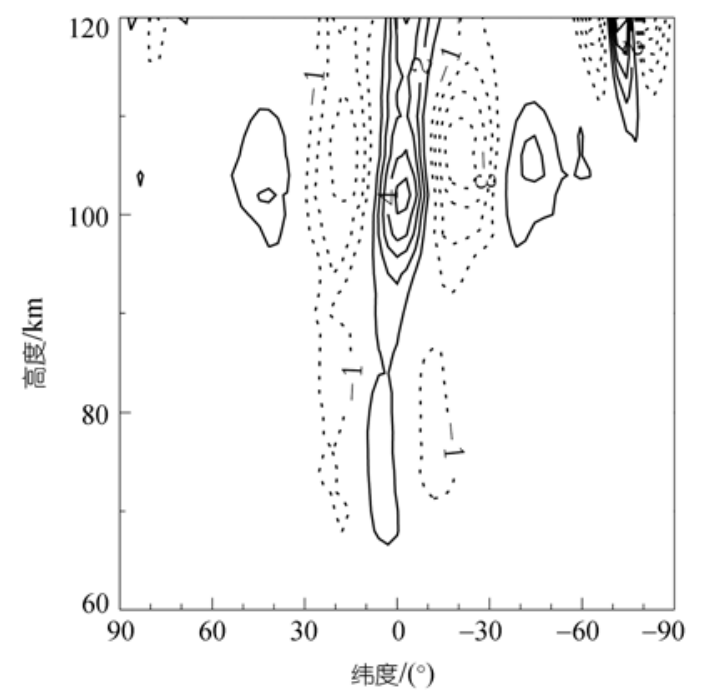

图 5 经过了 $18 \mathrm{~h}$ 的非线性传播后, 周日潮汐波导致的背 景温度的变化

用. 背景温场的改变最大可达 $10 \mathrm{~K}$, 即在一月份周日 潮汐波对背景的平均加温最大可达约 $13 \mathrm{~K} \cdot \mathrm{d}^{-1}$. 这 一结果说明周日潮汐的非线性传播也会强烈影响全 球中、高层大气的热力学暂态结构.

\section{2 半日潮汐}

采用我们的数值模式计算出半日潮汐在中、高层 大气中经过了 $6 \mathrm{~h}$ 的非线性传播后东向风振幅的纬度 和高度分布, 然后将计算结果减去图 1(b)中所示的 GSWM 的结果, 即可得到图 6. 也就是说, 图 6 中所 给出的是非线性传播 $6 \mathrm{~h}$ 后, 半日潮汐东向风振幅与
GSWM 结果的差异; 这一差异事实上也反映了半日 潮汐的短期变化. 这是首次给出半日潮汐在中、高层 大气中的全非线性传播结果. 图 6 中的实线表示正值, 虚线表示负值，等值线间的间隔为 $1.5 \mathrm{~m} / \mathrm{s}$. 由于 90 $\mathrm{km}$ 以下，计算结果和模式结果差异很小，为了放大 我们所感兴趣的区域, 图中只给出了 90 120 km 的高 度范围内它们的差异. 由图 6 可见, 非线性效应使东 向风潮汐振幅在有的区域增加, 有的区域减小, 并且 潮汐振幅(图 1 所示)越大、非线性效应越强的区域, 这种差异也越大. 在东向风潮汐幅度的两个极大值 (高度 $108 \mathrm{~km}$, 北纬 $48^{\circ}$ 和高度 $112 \mathrm{~km}$, 南纬 $51^{\circ}$ 处) 附近, 东向风潮汐变化也出现负的极值. 这些结果说 明, 非线性效应是导致大气潮汐短期变化的重要原 因, 它对大气潮汐的全球结构有显著作用.

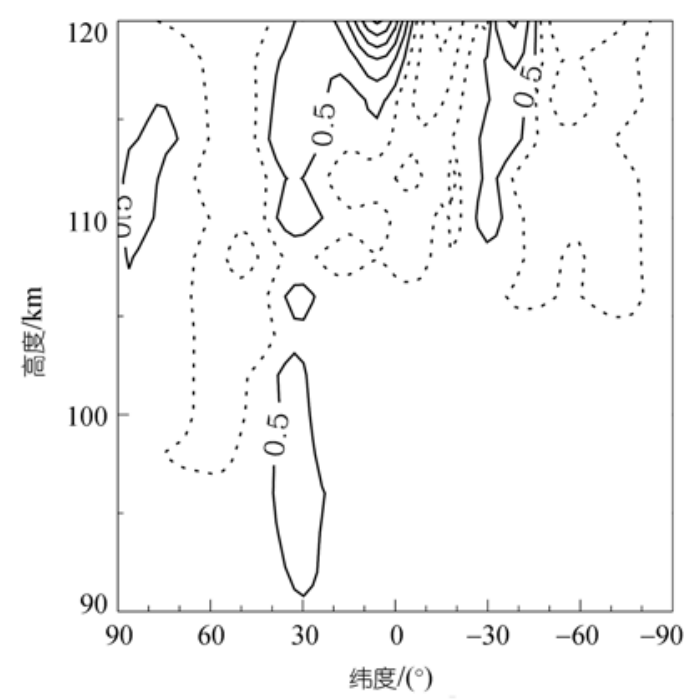

图 6 非线性传播 $6 \mathrm{~h}$ 后, 半日潮汐东向风振幅与 GSWM 结果的差

在非线性效应的作用下，潮汐波与背景大气的 非线性相互作用会改变背景大气的动力学和热力学 结构. 前面的计算结果已经清晰地展示出周日潮汐 的非线性传播会明显改变背景东向风场. 这里我们 给出半日潮汐非线性传播导致的背景东向风场变化 量的纬度和高度分布(图 7), 即(3)式中的 $\boldsymbol{g}_{0}(\varphi, r, t)$. 图中, 实线代表正值(即波导致的背景风场增强), 虚 线代表负值(即波导致的背景风场减弱)，等值线间的 间隔为 $1 \mathrm{~m} / \mathrm{s}$. 从图 7 可以观察到, 在 90 120 km 的高 度上, 即中层顶和低热层的范围内, 半日潮汐和背景 的非线性相互作用对几乎所有纬度上的背景风场都 有影响? 在北半球的中、低纬地区, 这种相互作用会 
对东向风背景风场产生一个减速效应, 最大可达-10 $\mathrm{m} / \mathrm{s}$; 相反, 在北半球的高纬和南半球的大部分地区 对东向背景风场则产生加速效应, 最大可达 $6 \mathrm{~m} / \mathrm{s}$. 这说明, 半日潮汐的非线性传播会影响全球中、高层 大气的动力学结构.

为了研究潮汐的非线性传播对中、高层大气热力 学结构的影响, 我们也给出了半日潮汐波导致的背 景温度变化(图 8). 图中, 实线代表正值(即波导致的 背景增温), 虚线代表负值(即波导致的背景降温), 等 值线间的间隔为 $2 \mathrm{~K}$. 由图 8 可见，周日潮汐和背景 的非线性相互作用会对赤道地区和南半球中纬地区 的背景产生明显的增温作用, 最大可达 $20 \mathrm{~K}$, 而在这 两个增温区之间以及北半球的大部分区域, 这种相 互作用会导致背景大气的降温，最大可达-12 K. 这 一结果说明半日潮汐的非线性传播也会强烈影响全 球中、高层大气的热力学暂态结构.

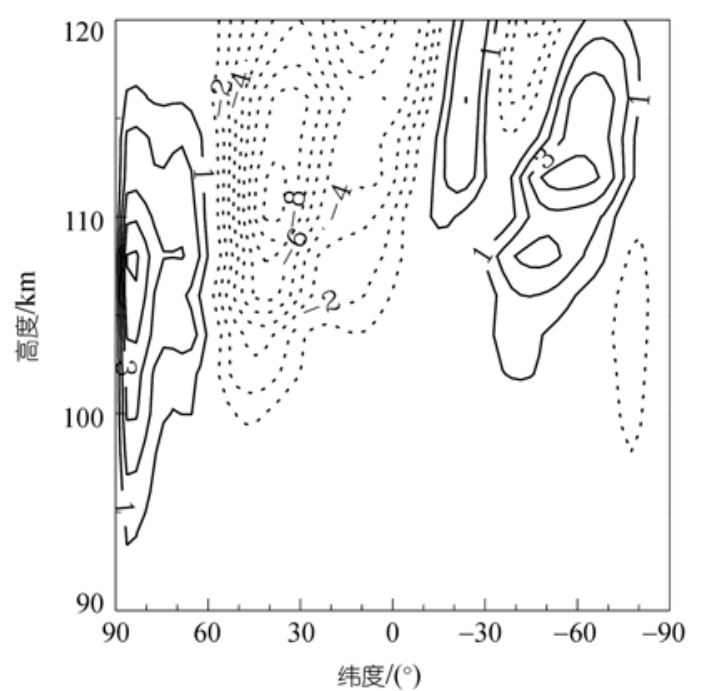

图 7 经过了 $6 \mathrm{~h}$ 的非线性传播后, 半日潮汐波导致的东向 背景风场变化

\section{3 讨论与结论}

以 GSWM 给出的一月份周日和半日潮汐线性稳 态解作为初值, 采用三维球坐标系下中层大气的全 非线性动力学数值模式, 模拟了从地面到低热层的 大气中, 周日和半日潮汐的线性和非线性传播, 并将 模拟结果与 GSWM 结果进行了定量比较. 研究结果 表明受非线性效应影响, 潮汐在中、高层大气中, 特 别是在中层顶和低热层高度上(潮汐振幅较大的区域)

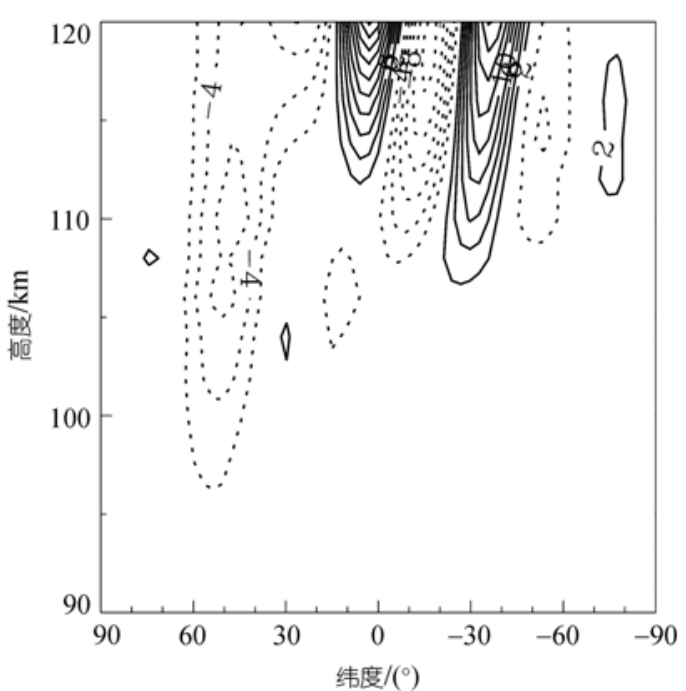

图 8 经过了 $6 \mathrm{~h}$ 的非线性传播后, 半日潮汐波导致的背景 温度变化

会呈现明显的短期变化. 这说明非线性是导致潮汐 短期变化的重要原因, 从而提示我们在中层顶和低 热层高度上研究大气潮汐时, 必须考虑非线性效应 的影响. 另外, 潮汐波与背景大气的非线性相互作用 会显著改变背景风场和温场, 并且背景大气的改变 依赖于潮汐振幅. 这表明潮汐与背景大气的相互作 用会对中、高层大气背景的动力学和热力学暂态结构 有重要影响, 伴随着潮汐的全球传播, 这种相互作用 会直接影响全球大气的动力学和热结构.

这是首次给出全球大气潮汐的非线性传播解, 计算结果很好地符合了 $\mathrm{Yi}^{[5]}$ 在极区的VHF雷达观测. 当然, 要想更细致地反映潮汐的短期变化, 在模式计 算中也必须考虑背景大气参数(如背景风场、温场、 源和耗散等)的短期变化. 但是考虑到背景大气参数 变化的时间尺度一般而言总是大于波周期，因而采 用月平均的背景大气参量应该还是一个合理的近似. 另外, 周日和半日潮汐都有明显的季节变化特征, 这 里我们虽然只给出了一月份的计算结果, 但是对其 它月份的计算都可得到相似的结果. 总体而言, 振幅 越大, 非线性效应越强. 在一月份的大部分空间区域, 半日潮汐的振幅要大于周日潮汐, 因而, 本文中非线 性导致的半日潮汐短期变化和背景大气变化都大于 周日潮汐相应的计算结果.

Zhang 等 ${ }^{[9]}$ 利用 MF 雷达研究了在冬季, 武汉 $\left(30^{\circ} \mathrm{N}, 114^{\circ} \mathrm{E}\right)$ 上空中层顶和低热层区域潮汐的基本 特征, 发现观测结果和GSWM的差异很大. 特别是观 
测结果表明在 $90 \mathrm{~km}$ 以上, 周日潮汐振幅会出现随高 度下降趋势, 这在GSWM中完全无法反映. 而利用我 们的全非线性模式的计算结果则清晰地显示出了这 一趋势, 这说明非线性效应可能是导致GSWM和观 测结果差异的重要原因.

由于GSWM本质上是一个线性模式，所以潮汐 与背景大气的非线性相互作用以及由此产生的背景 大气风场和温场变化在GSWM中无法反映. 我们的 非线性模拟结果则首次定量地显示出这种非线性相 互作用导致的背景大气变化. 我们注意到, 在一月份 半日潮汐的非线性传播在北半球的中纬地区会对东 向背景风产生强烈的减速作用(图 7), 并且在 $110 \mathrm{~km}$ 以下的高度, 这种减速效应随高度增加而增加, 意味 着潮汐的传播在一月份会导致北半球中纬地区东向 背景风场随高度的增加而下降, 这与Zhang等 ${ }^{[9]}$ 在武 汉冬季对背景风场的观测结果完全一致.

重力波在南、北半球传播的不对称性是近年来 中、高层大气研究的热点和前沿问题 ${ }^{[10]}$, 它的研究关 系到全球大气模式中重力波效应参量化的精度，甚 至直接影响全球大气模式的精度. 目前公认的机制 有两个: 一是太阳对地球大气加热的南、北半球不对 称性; 二是低层大气对太阳辐射吸收(即重力波的激 发源)的南、北半球不对称性. 2004 年, Zhang和 $\mathrm{Yi}^{\mathrm{i}} 11$ 通 过数值模拟方法证实重力波在南、北半球的自由传播 本身也是不对称的. 本文中, 我们的研究显示出尽管 GSWM给出的半日潮汐的振幅分布(即计算初值)具 有一定的对称性(图 1(b))半日潮汐非线性传播导致的 背景风场和温场改变量却呈现出显著的不对称性(图 7 和 8), 这说明潮汐的非线性传播也可能是导致重力 波传播南、北半球不对称的重要原因.

这里需要说明的是, 虽然 $8 \mathrm{~h}$ 潮汐是与周日与半 日潮汐相互关联的重要波动, 但在本文中没有给出 8 $\mathrm{h}$ 潮汐非线性传播的结果. 这一方面是由于 GSWM 模式中并未给出 $8 \mathrm{~h}$ 潮汐的稳态结果, 无法采用本文 的方法来获取其非线性传播解; 另一方面是由于与 周日与半日潮汐相比, $8 \mathrm{~h}$ 潮汐的幅度较小, 其非线 性传播对潮汐和背景的短期变化影响较小.
致谢 本工作为国家自然科学基金(批准号: 40336054, 40274051)、教育部新世纪优秀人才支持计划和武汉大学地 球空间环境与大地测量教育部重点实验室开放基金资助项 目.

\section{参考文献}

1 Chapman S, Lindzen R S. Atmospheric Tides. Dordrecht, Holland: D. Reidel, 1970

2 Hagan M E, Forbes J M, Vial F. A numerical investigation of the propagation of the quasi 2-day wave into the lower thermosphere. J Geophys Res, 1993, 98: 23193 23205

3 Hagan M E, Burrage M D, Forbes J M, et al. GSWM-98: Results for migrating solar tides. J Geophys Res, 1999, 104: $6813 \sim 6827[\mathrm{DOI}]$

4 Hagan M E, Forbes J M. Migrating and nonmigrating diurnal tides in the middle and upper atmosphere excited by tropospheric latent heat release. J Geophys Res, 2002, 107, 4754, doi:10.1029/2001JD001236

5 Yi F. Short-term variability and temporary structures of tides and mean wind in the polar summer mesosphere. J Atmos Sol-Terr Phys, 2001, 63: 749 757[DOI]

6 Grieger N, Volodin E M, Schmitz G, et al. General circulation model results on migrating and nonmigrating tides in the mesosphere and lower thermosphere, Part I: comparison with observations. J Atmos Sol-Terr Phys, 2002, 64: 897 911[DOI]

7 黄春明, 易帆, 张绍东. 利用谱方法数值求解三维球坐标系下 的 Navier-Stokes 方程. 空间科学学报, 2005, 25(1): 37 43

8 Forbes J M. Atmospheric tides, 1. Model description and results for the solar diurnal component. J Geophys Res, 1982, 87: 5222 5240

9 Zhang S D, Yi F, Hu X. MF radar observation of mean wind and tides of winter mesopause $(80 \sim 98 \mathrm{~km})$ region over Wuhan $\left(30^{\circ} \mathrm{N}\right.$, $114^{\circ}$ E). J Atmos Sol-Terr Phys, 2004, 66(1): 15 25[DOI]

10 Siskind D E, Eckermann S D, McCormack J P. Hemispheric differences in the temperature of the summertime stratosphere and mesosphere. J Geophys Res, 2003, 108: ACL10-1-10-13

11 Zhang S D, Yi F. A numerical study on global propagations and amplitude growths of large scale gravity wave packets. J Geophys Res, 2004, 109, D07106, doi:10.1029/2003JD004429

(2004-12-30 收稿, 2005-04-20 收修改稿) 